\title{
DIABETES MELLITUS: AVALIAÇÃO E CONTROLE ATRAVÉS DA GLICEMIA EM JEJUM E HEMOGLOBINA GLICADA
}

\section{DIABETES MELLITUS: AVALIATION AND CONTROL THROUGH FASTING GLUCOSE AND GLYCATED HEMOGLOBIN}

\author{
Rodrigo Cimino Sá1 \\ Edna Aparecida Ferraz de Araújo Navas² \\ Silvio Rubens Alves ${ }^{3}$
}

Resumo: O Diabetes mellitus é desencadeado quando a produção ou ação de insulina tornam-se deficientes gerando sintomas e complicações crônicas sérias, tratando-se atualmente de um problema de saúde pública mundial. O objetivo foi realizar estudo das concentrações séricas das glicemias em jejum, A1C e estimativas dos níveis de glicose média baseados nos valores da A1C de pacientes diabéticos atendidos em um laboratório do município de São José dos Campos-SP. Durante o período de setembro a outubro de 2012, foram analisados os resultados dos exames de glicemia em jejum e A1C de 3.149 pacientes na faixa etária entre 30 a 69 anos, e com base nessas informações calculou-se a glicose média estimada de cada paciente através da equação: $28,7 \times A 1 C$ - 46,7. Pacientes não-diabéticos foram 62,2\%, de risco ou pré-diabéticos $11,2 \%$ e diabéticos 26,6\%. Discussão. No grupo de diabéticos, os valores da glicemia em jejum, A1C e glicose média calculada se correlacionaram, demonstrando que quanto maiores as taxas da glicemia em jejum, maior será a estimativa da glicose média e, consequentemente, o nível de A1C. A média dos níveis glicêmicos entre 100 a $125 \mathrm{mg} / \mathrm{dL}$ deve ser uma meta aos pacientes diabéticos, condição fundamental na prevenção de riscos e complicações inerentes à doença.

Palavras-chave: Diabetes Mellitus; glicemia em jejum; glicose média estimada; hemoglobina glicada.

Abstract: Diabetes mellitus is triggered when the production or action of insulin become disabled causing serious symptoms and chronic complications; therefore, it is currently a public health problem worldwide. Objective. Conduct a study of serum concentrations of fasting glucose, A1C, and estimated average glucose levels based on the values of $A 1 C$ for diabetic patients seen at a laboratory in São José dos Campos, Brazil. During the period from September to October 2012, test results of fasting glucose and A1C of 3,149 patients 30-69 years old were analyzed. Based on this information, we calculated the estimated average glucose of each patient through the equation: $28.7 \times A 1 C$ - 46.7. Non-diabetic patients were $62.2 \%$, risk or pre-diabetics $11.2 \%$, and diabetics $26.6 \%$. In the diabetic group, the values of fasting plasma glucose, $A 1 C$, and calculated average glucose were correlated, showing that the higher the fasting glucose, the greater the estimated average glucose and consequently the level of A1C. The mean glucose levels between 100 to $125 \mathrm{mg} / \mathrm{dL}$ should be a goal for patients with diabetes, a condition essential to prevent risks and complications inherent in the disease.

Keywords: diabetes mellitus; fasting glucose; estimated average glucose; glycated hemoglobin.

\footnotetext{
1 Graduado em Biomedicina - Universidade do Vale do Paraíba - Univap. E-mail: ddigocimino@hotmail.com.

2 Doutora em Biopatologia Bucal - Universidade Estadual Paulista Júlio de Mesquita Filho - UNESP. E-mail: edna.navas@hotmail.com.br.

${ }^{3}$ Graduado em Ciências Biológicas - Univap e Supervisor técnico do Valeclin Laboratório de Análises Clínicas. E-mail: supervisao@valeclin.com.br.
} 


\section{INTRODUÇÃO}

O Diabetes mellitus é uma doença desencadeada quando se tem uma produção ou ação de insulina deficiente levando a sintomas agudos e, consequentemente, a complicações crônicas características (CZEPIELEWSKI, 2001).

O distúrbio é movido pelos metabolismos da glicose, gorduras e proteínas, apresentando graves consequências, indiferente se surgem abruptamente ou lentamente. Atualmente, é constituído em problema de saúde pública de esfera mundial, pela quantidade de pessoas diagnosticadas com a doença, principalmente no Brasil, apresentando a maior prevalência (7,6\%) nos indivíduos com faixa etária entre 30 a 69 anos (CZEPIELEWSKI, 2001; GROSS et al., 2002).

O diagnóstico do diabetes baseia-se, fundamentalmente, nos valores da glicemia plasmática de jejum (8 horas) ou após uma ingestão acentuada de glicose por via oral, ou ainda pelo nível de hemoglobina glicada, este ainda muito eficaz e solicitado pelos médicos no acompanhamento dos pacientes já diagnosticados com a doença (GROSS et al., 2002).

Quando dosada a glicemia em jejum dos pacientes, o resultado apresentado fornece apenas o indício de como está o nível da glicose plasmática nas últimas horas, ao contrário da dosagem da hemoglobina glicada (A1C), fundamental no monitoramento da doença, uma vez que avalia a média glicêmica do paciente nos últimos 3 meses (PINHEIRO, P. 2010). Manter o nível de A1C inferior a $7 \%$ é uma das principais metas para o bom controle da doença. Estudos mostram que as complicações crônicas decorrentes do diabetes começam a se manifestar quando os níveis de A1C apresentam-se constantemente acima de $7 \%$ (ANDRIOLO et al., 2009).

O exame de glicemia em jejum é o meio clássico de se diagnosticar o diabetes mellitus. Considerando que quando em jejum, a taxa de glicose circulante no sangue deve situar-se abaixo de $100 \mathrm{mg} / \mathrm{dL}$ nos pacientes considerados normais e, quando esta encontrar-se entre 100 e 125 mg/dL, significa que o paciente apresenta alteração na glicemia em jejum, também denominada hiperglicemia não diabética ou pré-diabetes. A definição pré-diabetes baseia-se no fato de que 1 a cada 4 pacientes com alteração na glicemia de jejum, desenvolverão critérios para o desencadeamento do diabetes mellitus dentro de 3 a 5 anos, e, ainda, se o paciente apresentar outros fatores de risco como obesidade e histórico familiar, o risco tornase ainda maior (PINHEIRO, 2010).

Quando o valor da glicemia em jejum apresenta-se acima de $125 \mathrm{mg} / \mathrm{dL}$ em pelo menos duas coletas sanguíneas realizadas em momentos diferentes, tem-se o diagnóstico fidedigno para o diabetes mellitus (PINHEIRO, 2010).

As hemácias, cuja função é transportar oxigênio por todos os tecidos do corpo humano, são constituídas, principalmente, por hemoglobina (DE CAMPOS, 2006). Quando os níveis da glicemia sanguínea encontram-se aumentados, uma parcela da hemoglobina começa a ligar-se a essa glicose circulante em excesso, produzindo a hemoglobina glicada, ou seja, hemoglobina ligada à glicose, sendo essas acumuladas no interior das hemácias, adquirindo, com isso, uma meia-vida a partir da delas (PINHEIRO, 2010; SACKS, 2006). O tempo em que 
as hemácias ficam expostas à glicose no sangue é igual ao seu tempo de vida, de 3 a 4 meses, o que resulta na reflexão da hemoglobina glicada na glicemia média dos últimos $60 \mathrm{a}$ 120 dias que antecedem o exame (PINHEIRO, 2010).

É fundamental que se realize o exame de $\mathrm{A} 1 \mathrm{C}$, com frequência, em todos os pacientes diagnosticados com diabetes, com o objetivo de documentar o andamento do controle glicêmico na sua fase avaliativa inicial e, subsequentemente, como parte do acompanhamento contínuo do paciente (ANDRIOLO, 2009).

Por tradição, a A1C representa a média geral das glicemias médias diárias, incluindo as glicemias colhidas em jejum e pós-prandiais dos últimos dois e três meses. Quando, na realidade, o processo de glicação da hemoglobina acontece durante todo o tempo de vida do glóbulo vermelho, por aproximados 4 meses. Porém, durante esses 4 meses, as glicemias colhidas recentemente são as que mais influenciam o valor da A1C (ANDRIOLO et al., 2009). De fato que, estudos teóricos e clínicos determinam que um paciente estavelmente controlado apresente sua A1C distribuída da seguinte maneira: $50 \%$ formada no mês precedente ao exame, $25 \%$ no mês anterior a este e os outros $25 \%$ restantes 90 ou 120 dias antecedentes ao exame (CHANDALIA; KRISHNASWAMY, 2002).

Os níveis de $\mathrm{A} 1 \mathrm{C}$, diferente da glicemia, não voltam ao normal logo após a normalização desta na corrente sanguínea. O tempo necessário para a A1C alcançar os níveis esperados após um caso de descontrole são de, aproximadamente, 3 meses (ANDRIOLO; SUMITA, 2006).

Com o passar dos anos, a hiperglicemia prolongada desencadeou o desenvolvimento de lesões orgânicas extensas e irreversíveis que causavam danos aos olhos, aos rins, aos nervos, aos pequenos e grandes vasos, bem como a coagulação sanguínea (MIRANDA, G. 2004).

O diabetes pode ser prevenido, inicialmente, por meio de mudanças na rotina de vida, conforme demonstram importantes estudos conduzidos ao longo do mundo, que provam que hábitos de vida mais saudáveis, como uma dieta balanceada, rica em fibras, em busca do peso corporal adequado, associada à prática de exercícios físicos de, pelo menos, 150 minutos semanais, são eficazes, nos indivíduos pré-diabéticos, para reduzir o risco de desenvolvimento do diabetes mellitus em $58 \%$ e, nos indivíduos diabéticos, de reduzir até 2,0 \% o nível de A1C, de acordo com os resultados do estudo conduzido pelo DSP - Finnish Diabetes Prevention Study Group (TUOMILEHTO et al., 2001; NATHAN et al., 2009).

Em meados de 2008, baseado nos resultados obtidos com o estudo ADAG (A1C Derived Average Glucose Study Group), foi publicado um trabalho, no qual se relacionaram os valores dos níveis de $\mathrm{A} 1 \mathrm{C}$ com seus respectivos níveis de glicose em jejum, passando a ser usado, gradativamente, entre os médicos e diabéticos o termo glicose média estimada, cuja função é de complementar a informação clínica do paciente, mediante o resultado da A1C. Com o surgimento do conceito glicose média estimada, os resultados que, até então, eram expressos em termos de percentual de A1C, passaram a ser expressos em $\mathrm{mg} / \mathrm{dL}$ (ANDRIOLO et al., 2009; NATHAN et al., 2008; SUMITA, 2009). 
A substituição do valor da A1C em glicose média facilita a interpretação relacionada ao controle e monitoramento do diabetes juntamente ao risco do surgimento de complicações crônicas (NATHAN et al., 2008; SUMITA, 2009). Esse valor é obtido por meio da equação matemática desenvolvida pelo grupo de estudo (ADAG), em que a glicose média estimada $(\mathrm{mg} / \mathrm{dL})$ é igual a 28,7 $\times \mathrm{A} 1 \mathrm{C}-46,7$. A partir dos níveis da A1C é possível fazer uma estimativa da taxa média de glicose dos últimos 3 meses, seguindo a equação, por exemplo, se o nível de A1C for de $6 \%$, a glicose média estimada, já com variação, será de 126 (100-152) mg/dL (SACKS, 2008; SUMITA, 2009; PINHEIRO, 2010).

O objetivo foi realizar estudo das concentrações séricas da glicemia em jejum, da A1C e da estimativa dos níveis de glicose média baseados nos valores da hemoglobina glicada de pacientes diabéticos atendidos em um laboratório do município de São José dos Campos SP.

\section{METODOLOGIA}

Obtiveram-se os valores dos exames de hemoglobina glicada e glicemia em jejum dos pacientes com faixa etária entre 30 a 69 anos, atendidos em um laboratório de análises clínicas que presta atendimento à rede pública e privada no município de São José dos Campos - SP. Esses dados foram obtidos entre o período de outubro a setembro de 2012 com casuística representada conforme Tabela 1:

Tabela 1 - Total de pacientes cujos resultados dos exames de A1C e glicemia em jejum foram obtidos para o estudo

\begin{tabular}{cc}
\hline Total de Pacientes & $\mathbf{3 . 1 4 9}$ \\
\hline Sexo masculino & 1.170 \\
Sexo feminino & 1.979 \\
\hline
\end{tabular}

Com base nas informações relativas às concentrações séricas da glicemia em jejum e A1C, foi possível calcular, com base no valor da $A 1 C$, no programa Office Excel $\AA_{\text {, a glicose }}$ média estimada de cada paciente, por meio da equação: $28,7 \times A 1 \mathrm{C}-46,7$, e excluir do estudo os pacientes não diabéticos, bem como separar os de risco ou pré-diabéticos dos diabéticos propriamente ditos.

Na pesquisa, foram utilizadas apenas informações referentes às dosagens de glicemia em jejum, A1C, cálculo da glicose média pela A1C, e informações referentes ao sexo dos pacientes, no intuito de se preservar as suas identidades e da instituição concedente.

Os resultados dos níveis de glicemia em jejum, A1C e consequente estimativa da glicose média foram relacionados e analisados por testes paramétricos, expressos em percentual e apresentados na forma de tabelas e gráficos, utilizando-se o software Excel®, propiciando uma visão geral sobre o conjunto dos dados e determinando a importância que tem os exames de hemoglobina glicada e glicemia em jejum sobre a avaliação e controle do diabetes mellitus. 


\section{RESULTADOS}

Analisando os dados, foi possível excluir do estudo o grupo dos pacientes nãodiabéticos, ou seja, com os valores de glicemia em jejum abaixo de $100 \mathrm{mg} / \mathrm{dL}$ e nível de A1C menor que $6,0 \%$, bem como separar o grupo de pacientes de risco ou pré-diabetes, com glicemia em jejum entre 100 e $125 \mathrm{mg} / \mathrm{dL}$ e A1C entre 5,7 e 6,4\% do grupo de pacientes diagnosticados com diabetes, cujos valores da glicemia em jejum apresentou-se acima de 125 $\mathrm{mg} / \mathrm{dL}$ e A1C acima de 6,4\%. Esses números são representados na Tabela 2 e no Gráfico 1:

Tabela 2 - Número (n) e porcentagem (\%) dos pacientes não-diabéticos, pré-diabéticos e diabéticos

\begin{tabular}{ccc}
\hline Grupos & $\mathbf{n}$ & $\%$ \\
\hline Não-diabéticos & 1.961 & 62,2 \\
de Risco (Pré-diabéticos) & 352 & 11,2 \\
Diabéticos & 836 & 26,6 \\
\hline
\end{tabular}

\section{Gráfico 1 - Representação percentual da população de estudo: Pacientes não- diabéticos, pré-diabéticos e diabéticos}

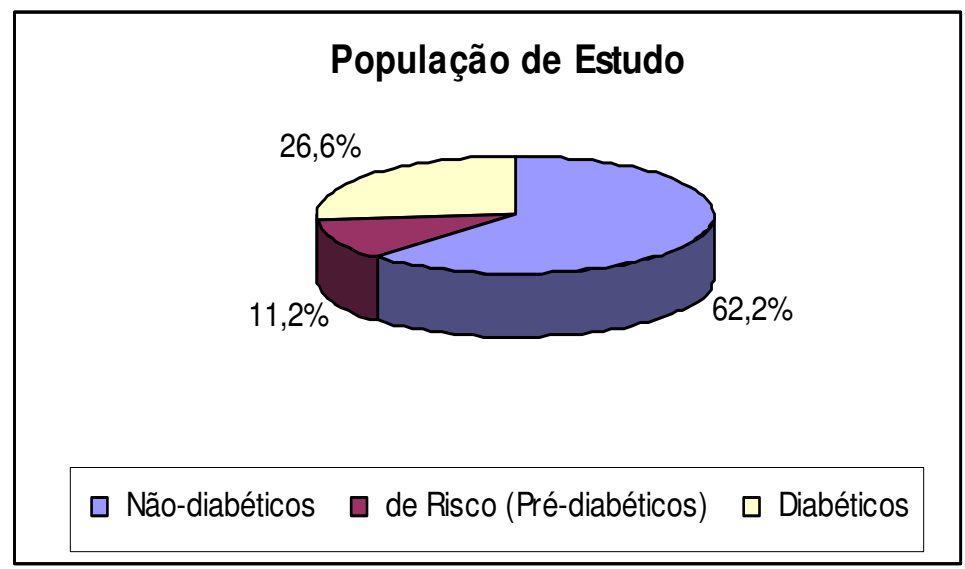

Os pacientes de maior interesse, neste presente estudo, ou seja, os diabéticos $(26,6 \%)$ tiveram seus valores de glicemia em jejum, A1C e glicose média relacionados, os quais são representados nos Gráficos 2 e 3. 
Gráfico 2 - Relação dos valores de A1C com a glicemia em jejum obtida dos pacientes diabéticos

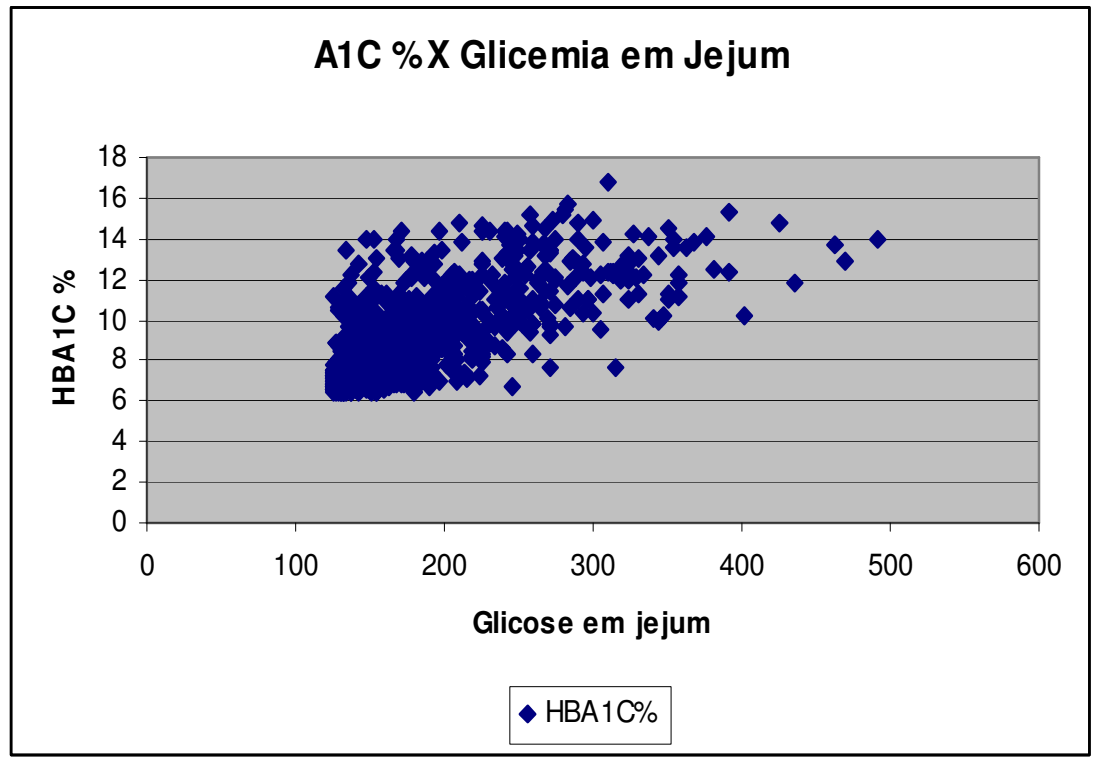

\section{Gráfico 3 - Relação dos valores da glicemia em jejum com a estimativa da glicose média obtida dos pacientes diabéticos}

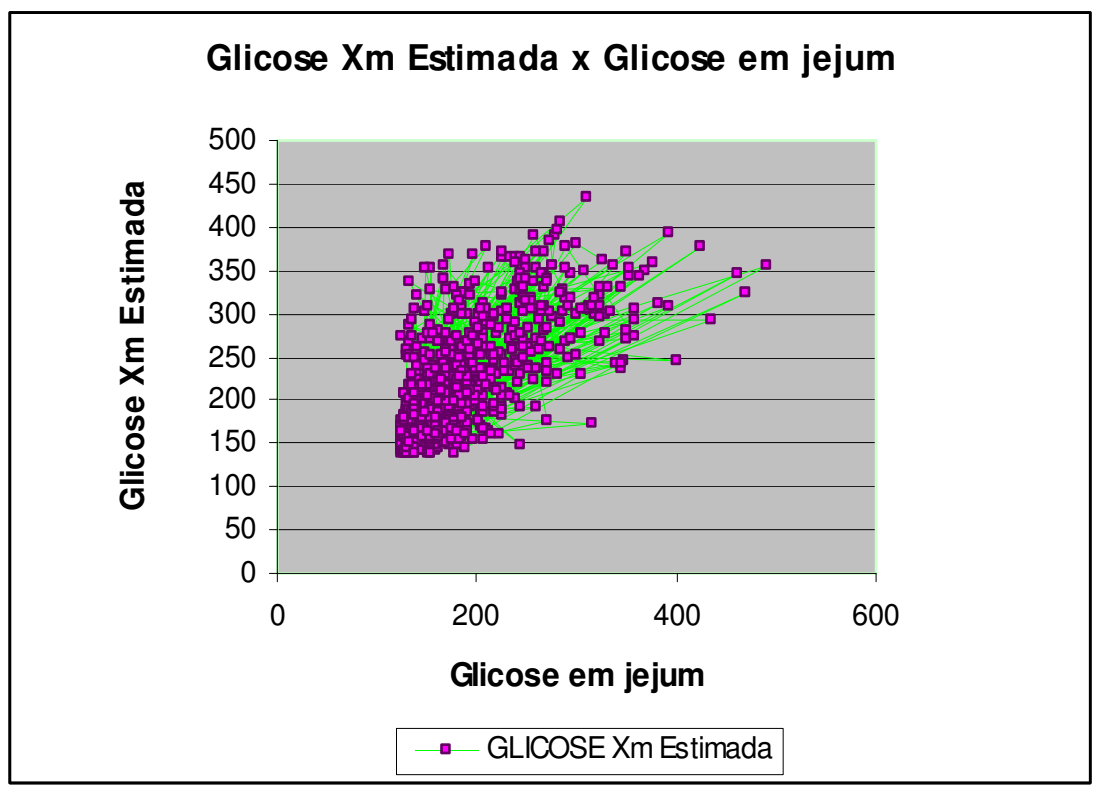

Como análises finais foram calculadas as médias $(\mathrm{Xm})$ dos valores da glicemia em jejum, A1C e estimativa da glicose média dos pacientes diabéticos, e os resultados encontrados são representados conforme a Tabela 3: 
Tabela 3 - Representação das médias (Xm) da glicemia em jejum, A1C e glicose média dos pacientes diabéticos

\begin{tabular}{lc}
\hline & Média (Xm) \\
\hline Glicemia em jejum & $121 \mathrm{mg} / \mathrm{dL}$ \\
A1C \% & $6,9 \%$ \\
Glicose média estimada & $151 \mathrm{mg} / \mathrm{dL}$ \\
\hline
\end{tabular}

\section{DISCUSSÃO}

Da totalidade de pacientes $(n=3.149)$, atendidos entre os meses de setembro à outubro de 2012, nesse laboratório do município de São José dos Campos - SP, foram utilizados os resultados dos exames de glicemia em jejum e hemoglobina glicada (A1C\%) para análise do estudo que, depois de concluída, foi constatada uma estatística uniforme, apresentada no gráfico 1, sendo $62,2 \%$ o número de indivíduos não-diabéticos, $11,2 \%$ os de risco com propensão a desencadear o diabetes futuramente se medidas preventivas não forem adotadas, e $26,6 \%$ os diabéticos. Essas proporções são consideradas satisfatórias e positivas, quando visualizado o número total de pacientes, qualificando-os como pessoas conscientizadas e preocupadas em manterem-se saudáveis.

Os $26,6 \%$ dos pacientes, os diabéticos em si formados em número por 836 indivíduos, tiveram seus valores de glicemia em jejum relacionados aos respectivos valores de A1C expressos no Gráfico 2 de dispersão através do software Excel® e, ao analisar o gráfico observa-se que quanto maior o valor da glicemia em jejum do paciente, consequentemente maior é o valor da hemoglobina glicada (A1C), mostrando a relação existente entre esses dois parâmetros. O valor de glicemia em jejum mais alto foi $491 \mathrm{mg} / \mathrm{dL}$ e A1C 16,76\%.

Outro parâmetro desses pacientes, também relacionado, foi o valor da glicemia em jejum com suas respectivas glicoses médias estimadas, expresso no Gráfico 3, também de dispersão, mostrando a proporção também existente entre esses dados, que quanto maior os valores da glicose em jejum do paciente, maior será a estimativa da sua glicose média, sendo a maior obtida igual a $434,3 \mathrm{mg} / \mathrm{dL}$.

Como análise comprobatória final, foi calculada a média $(\mathrm{Xm})$ da glicemia em jejum, A1C e glicose média estimada dos 836 pacientes, representados na tabela 3, confirmando a importância que se tem em manter a avaliação e controle dos níveis glicêmicos rotineiramente para que estes se reflitam positivamente no valor da $\mathrm{A} 1 \mathrm{C}$, eliminando riscos e complicações futuras, provenientes do mau controle da doença. As médias obtidas foram glicemia em jejum (121 mg/dL), A1C (6,9\%) e glicose média estimada (151 mg/dL), confirmando o que já foi dito, que quando se mantem um bom controle dos níveis glicêmicos, automaticamente é mantido um bom controle do nível de A1C.

Dos 836 pacientes, verificou-se que são poucos os conscientes que fazem o correto controle da taxa glicêmica, uma vez que, deste total, apenas 110 apresentaram taxa de hemoglobina glicada (A1C) abaixo de 7,0\%. 
De acordo com Pinheiro (2010), quando os níveis de A1C encontram-se estacionados acima dos 7,0\% durante um longo período, esses pacientes ficam susceptíveis a um maior risco de apresentarem complicações crônicas, como doenças cardiovasculares, renais, dos nervos periféricos, dos olhos ou, até mesmo, a amputação parcial ou total de membros, entre outros problemas, dependendo do ponto atingido pelo descontrole do paciente.

Apenas para constatação da importância que o exame de $\mathrm{A} 1 \mathrm{C}$ tem no acompanhamento do paciente diabético, exemplificamos, aqui, o caso de um paciente passa os últimos 3 meses sem dieta controlada e usando os medicamentos para o diabetes de modo irregular, mas 24 a 48 horas antes da realização dos exames, resolve tomar os medicamentos de modo correto. Quando for dosada a sua glicemia em jejum é, possível que esta se encontre dentro ou próximo da normalidade, dando a falsa ideia de que seu diabetes está bem controlado. Porém, se a A1C também for dosada, esta estará, visivelmente, alterada, indicando que, na verdade, o diabetes não está sendo tratado como deveria.

Apesar de ainda não ser mundialmente aceito, já há grupos que usam a $\mathrm{A} 1 \mathrm{C}$ também para diagnosticar o diabetes mellitus. Duas coletas diferentes com valores acima de $6,5 \%$ seriam suficientes para se fechar o diagnóstico.

\section{CONCLUSÃO}

Manter a média dos níveis glicêmicos entre 100 a $125 \mathrm{mg} / \mathrm{dL}$ deve ser uma meta de todos pacientes portadores de diabetes, uma vez que a glicose média entre esses valores assegura a dosagem da hemoglobina glicada (A1C) sempre abaixo de 7,0\%, fundamental na prevenção de riscos e complicações inerentes à doença.

O exame de $\mathrm{A} 1 \mathrm{C}$ desempenha excelente papel na avaliação e controle do diabetes, fornecendo a glicose média do paciente nos últimos 3 meses, sendo importante sua dosagem sempre nesse período. Já, o exame de glicemia em jejum não se aplica diretamente ao tratamento, porém apresenta potencial importância no diagnóstico, sendo o mais específico, uma vez que já é confirmado o diabetes se em duas dosagens realizadas em momentos distintos, o resultado apresentado for $>125 \mathrm{mg} / \mathrm{dL}$.

\section{REFERÊNCIAS}

ANDRIOLO, A. et al. Grupo interdisciplinar de padronização da hemoglobina glicada - A1C. Atualização sobre hemoglobina glicada (A1C) para avaliação do controle glicêmico e para o diagnóstico do diabetes: Aspectos clínicos e laboratoriais. Posicionamento oficial - 3.2009 ed. Disponível em: < http://www.saudedireta.com.br/docsupload/1332104952Hemoglobina\%20Glicada.pdf>. Acesso em: 15 ago. 2012.

ANDRIOLO, A.; SUMITA, N. M. Importância da determinação da hemoglobina glicada no monitoramento do paciente portador de diabetes mellitus. Jornal Brasileiro de Patologia e Medicina Laboratorial, v. 42, n. 3, 2006. Disponível em: 
$<$ http://www.scielo.br/scielo.php?script=sci arttext\&pid=S167624442006000-300002

\&lang=pt>. Acesso em: 15 ago. 2012.

CHANDALIA, H. B.; KRISH-NASWAMY, P. R. Glycated Hemoglobin. Current Science, v. 83, n. 12, pp. 1522-1532, 2002.

CZEPIELEWSKI, M. A. Diabetes. 2001. Disponível em: <http://www.abcdasaude.com.br/artigo.php?127>. Acesso em: 22 set. 2012.

DE CAMPOS, S. Diabete/ Diabetes - 0 que é a Hemoglobina Glicada. 2006. Disponível em: <http://drashirleydecampos.com.br/noticias/19375>. Acesso em: 14 ago. 2012.

GROSS et al. Diabetes Melito: Diagnóstico, Classificação e Avaliação do Controle Glicêmico. Arq. Bras. Endocrinol. Metab., v. 46, n. 1, fev. 2002. Disponível em: <http://www.scielo.br/pdf/abem/v46n1/a04v46n1.pdf>. Acesso em: 17 out. 2012.

MIRANDA, G. Hemoglobina Glicada: sua importância para a avaliação do controle glicêmico em pacientes com diabetes mellitus - aspectos clínicos e laboratoriais. 2004. Disponível em: <http://www.cssj.com.br/site2/english/informativos detalhes.asp?id=32>. Acesso em: 14 ago. 2012.

NATHAN, D. M. et al. Translating the A1C Assay Into Estimated Average Glucose Values. Diabetes Care, v. 31, pp. 1-16, 2008.

NATHAN, D. M. et al. Medical Management of in Type 2 Diabetes: A Consensus Algorithm the Initiation and Adjustment of Therapy. Diabetes Care, v. 32, pp. 193-203, 2009.

PINHEIRO, P. Diabetes Mellitus: Diagnóstico e Hemoglobina Glicosilada. 2010. Disponível em: <http://www.mdsaude.com/2010/06/glicemia-hemoglobina-glicosilada.html>. Acesso em: 14 ago. 2012.

SACKS, D. B. Carbohydrates. In:BURTIS, C. A.; ASHWOOD, E. R.; BRUNS, D. E. 4th ed. Tietz Textbook of Clinical Chemistry and Molecular Diagnostics. St. Louis: Elsevier Saunders, 2006. pp. 837-901.

Translating hemo-globin A1C into average blood glucose: implications for clinical chemistry. Clinical Chemistry, v. 54, pp. 1756-1758, 2008.

SUMITA, N. M. A hemoglobina glicada e o Laboratório clínico. Jornal Brasileiro de Patologia e Medicina Laboratorial, v. 45, n. 1, fev. 2009. Disponível em: <http://www.scielo.br/scielo.php?script=sciarttext\&pid=S167624442009000100003\&lang=pt> . Acesso em: 15 ago. 2012.

TUOMILEHTO, J. et al. for the Finnish Diabetes Prevention Study Group. Prevention of type 2 diabetes mellitus by changes in life style among subjects with impaired glucose tolerance. N. Engl. J. Med., v. 344, n. 18, pp. 1343-1350, 2001. 